\title{
CHARACTERIZING PROSTAGLANDIN E RECEPTOR ON EPITHELIAL- MESENCHYMAL TRANSITION AND METASTASIS IN TNBC
}

M. Siu, J. Ho, I. Chuek, J. Chen, A. Kwong*, V. Shin

Surgery, The University of Hong Kong, Hong Kong, Hong Kong

GOALS: Metastatic breast cancer is characterised by uncontrolled cell proliferation, epithelial-mesenchymal transition (EMT), and escape from apoptosis. Prostaglandin E receptors have been reported to play a role in breast cancer carcinogenesis, however, its contribution to EMT reversal is largely unknown. Specifically, prostaglandin E2 (PGE2)induced prostaglandin E (EP) receptors have been reported to be associated with human cancer carcinogenesis. This study aims to evaluate the role of EP2 receptor in metastatic xenograft model and delineate the mechanistic pathway involving EMT in TNBC.

METHODS: We used a metastatic xenograft model of human breast cancer to study the role of EP receptor during cancer progression. In addition, construction of stable EP2expression MB-231 cells was used to study tumorigenesis and characterization of EP2 receptor. The functional role of EP2 receptor on cell proliferation, migration, invasion and EMT gene expression were examined in EP2-overexpressing and EP2 siRNA transfected cells. The xenografts were immunostained to examine the expression of Ki67 and CD31 in xenografts and the localization of EMT markers was assessed by immunofluorescence. RESULTS: Expression of EP2 receptor was induced during tumor progression and was found to be highly expressed in primary breast tumor tissues. The MB-231-EP2 clone developed a more aggressive tumor with larger tumor size, promoted metastasis, and resulted in a poorer survival. Cell proliferation (Ki-67) and angiogenesis (CD31) were more prominent in the metastatic than local xenografts. In addition, loss of E-cadherin and increase Twist expression were seen in the metastatic xenograft. In vitro study showed that MB-231-EP2 clone was more resistance to staurosporine-induced apoptosis than parental MB-231 cells. EP2 receptor promoted cell migration, and invasion were increased through modulation of EMT markers (E-cadherin, TWIST, ZEB1, MMP2). Immunofluorescence study demonstrated that E-cadherin was restored and Twist translocation was blocked in EP2 siRNA transfected cells, implicating silencing of EP2 receptor could reduce metastatic potential of the cells. Moreover, Twist expression level was higher in plasma of breast cancer patients than healthy controls. CONCLUSION: These novel findings suggest that EP2 receptor plays an important role in metastatic breast cancer though EMT. Targeting EP2 receptor could be a potential therapeutic strategy to improve survival in breast cancer patients. 